\title{
A BIM-Info Delivery Protocol
}

Martin Hooper and Anders Ekholm, (Lund University, Sweden)

\begin{abstract}
Today, with many of the technological issues of integrated information management resolved (perhaps excluding the matter of interoperability), defining the content and status of BIM information deliveries remains both a practical and a theoretical problem. New BIM tools and new design processes and procedures have led to a certain confusion of what information is needed for particular BIM uses. This paper seeks to explore and enable a method of defining the content of model information deliverables through a review of 2 key primary specific BIM uses: 3d Design Coordination and Early Energy Appraisal through an analysis of practical application. The scope of this study is limited to a review of information flow within residential projects in a Swedish context and looks at two projects with a view to identify and establish a common definition of the key BIM objects and properties necessary for particular tasks. The key deliverable from this study is the BIM-Info Delivery Protocol (IDP) which attempts to align consultant BIM-information delivery expectations and represents a tangible solution to assist consultants to manage BIM information. Concluding reflections consider the positioning of the IDP relative to the on-going development of IDMs / MVDs and highlight the key constituent parameters of an Information Delivery Specification (IDS).
\end{abstract}

Keywords: BIM, Building Information Modelling, information exchange, model information content

\section{Introduction}

\section{Background to Study}

The context of the study is directed toward the Swedish Design-Construct-Operate (DCO) sector where the concept of Leveransspecifikationer (Delivery Specifications) is suggested within the national guidelines for digital information management: Bygghandlingar 90 Byggsektorns Rekommendationer för Redovisning av Byggprojekt - Digital Leveranser för Bygg och Förvaltning (Swedish Standards Institute, 2008). These guidelines recommend the use of delivery specifications to accompany exchanges in digital information at all stages of the design, construct and operate process. However, there is a lack of concrete advice on how to develop information delivery specifications for defining and recording BIM information content in connection to or supporting a project based strategic BIM Implementation Plan.

While information is not lacking, practical experience in moving forward with BIM beyond office boundaries in Sweden still is in its infancy and there is hitherto an absence of developed examples of delivery specifications to accompany national standards (Ekholm et al., 2010).

\section{Goals and Research Questions}

This study has two aims. The first is to explore and enable a method of defining the content of model information deliverables through a review of two key primary specific BIM uses: $3 d$ Design Coordination and Early Energy Appraisal through an analysis of practical applications. The second is to design an associated process that ensures integration of information deliveries into a project plan, thereby securing greater certainty and efficiency of information exchanges. 
The central research questions for these aims are as follows:

$\downarrow$ Can BIM information delivery content be articulated in a commonly understood manner on a project basis?

\& Can a standard matrix be established that can be used for various BIM-Uses at various project stages that would help align information delivery expectations?

To answer these research questions five questions need to be answered:

\& What BIM information is needed at what time to enable efficient BIM Discipline Authoring toward rapid 3d Design Coordination at the Design Development Stage?

$\downarrow$ What BIM information is not needed? - Clarity is needed on what BIM information is not relevant at particular stages.

$\uparrow$ What level of detail is needed to carry out BIM-Uses at various stages?

$\rightarrow$ Is there a logical information order?

$\downarrow$ What is the logical information order of authoring BIM information for early Energy Analysis when it comes to generating BIM objects?

\section{Problem Status}

Whilst organisations such as the buildingSMART Alliance are investing considerable resources towards developing $A E C$ industry standardization in information exchanges, until understanding, control and trust can be gained in the use of IFC, IDM and MVD by DCO participants, a simpler system of describing information content requirements is essential for BIM implementation today on a consultant practice level.

Without a straightforward way of creating project specific IDM's, a clear understanding of the content of MVD's, and the ability to control information flow through such methods, trust may wither and die. Thus the driver for this study: the urgent need for a simple, user-friendly method of describing, in a commonly understood way, information deliverables.

\section{Hypothesis}

Perhaps a simpler method of generating IDM's and MVD's using commonly available tools within organisations would have greater mileage than those developed by external agents. Here it is conjectured that, as a valid alternative, BIM-Info Deliveries developed by architects and engineers for architects and engineers may offer the control, confidence and simplicity necessary for effective information exchange success.

In order for industry professionals to get the best out of BIM tools and work efficiently, one needs a big picture understanding of information need, honing individual tools and processes towards greater efficiency and certainty. Key to doing this is a thorough documentation of one's own business processes - use of a BIM-Info Deliveries Protocol as part of a BIM-Implementation Plan may offer an immediate and tangible solution.

\section{Methods}

\section{Research Design}

The research design has been flexible from the start to enable the framework of reference to emerge during the study. A literature review - including national standards guidelines such as CRC (2009) and industry press - together with consultant interviews and discussions revealed a real need for the development of BIM information delivery specifications to support cross-discipline communication and downstream use of data. To move forward, two case projects were identified. The first was to enable a closer examination of the issues involved. The second was to test a protocol proposal and enable categorization the building parts and level of detail required for set exchanges against a specific BIM-Use. 
The case study's strength is its ability to deal with a full variety of evidence - documents, interviews, and observation - beyond what might be available through other research approaches (Yin, 2003). Here, two important sources of evidence are direct observation and systematic interviewing.

Through its natural setting, the case study provides an ideal practical real-life context and a suitable grounded platform to consider and test strategic decisions with regard to information exchanges thereby creating an opportunity to introduce a move towards a greater clarity of purpose and improved efficiency in information sharing (Robson, 2002).

\section{Data Collection and Case Description}

The collection of empirical material has been assembled to firstly facilitate a deeper understanding of the circumstances and context of the information exchanges necessary to implement the said BIM-Uses and secondly to study the BIM-Content to be exchanged at object-level. To meet these objectives a series of design coordination workshops where carried out in connection with a (real world) residential case study project (Koggens Gränd) facilitating collection of empirical data, together with the generation and examination of a controlled experimental model (KonsultHus). The known characteristics of this first case fit well with the opportunities to break new ground and strive towards improvements in information management and uncovered a raft of issues to address. The second case enabled the extraction of more detailed information to populate the delivery specification.

\section{Case study 1: Koggens Gränd - Malmö's first owner-occupier flats}

Located in the Västra Hamnen area, Malmö, Koggens Gränd is an innovative new residential block with occupancy expected through 2012. As part of a larger development incorporated in the regeneration of Västra Hamnen the scheme presents 31 owner-occupier flats of between 45-72m². Prior to May 1, 2009 in Sweden it was not possible to build - or to convert through renovation - owner-occupier flats and Koggens Gränd represents one of Sweden's first residential building containing owner-occupier units on a larger scale.

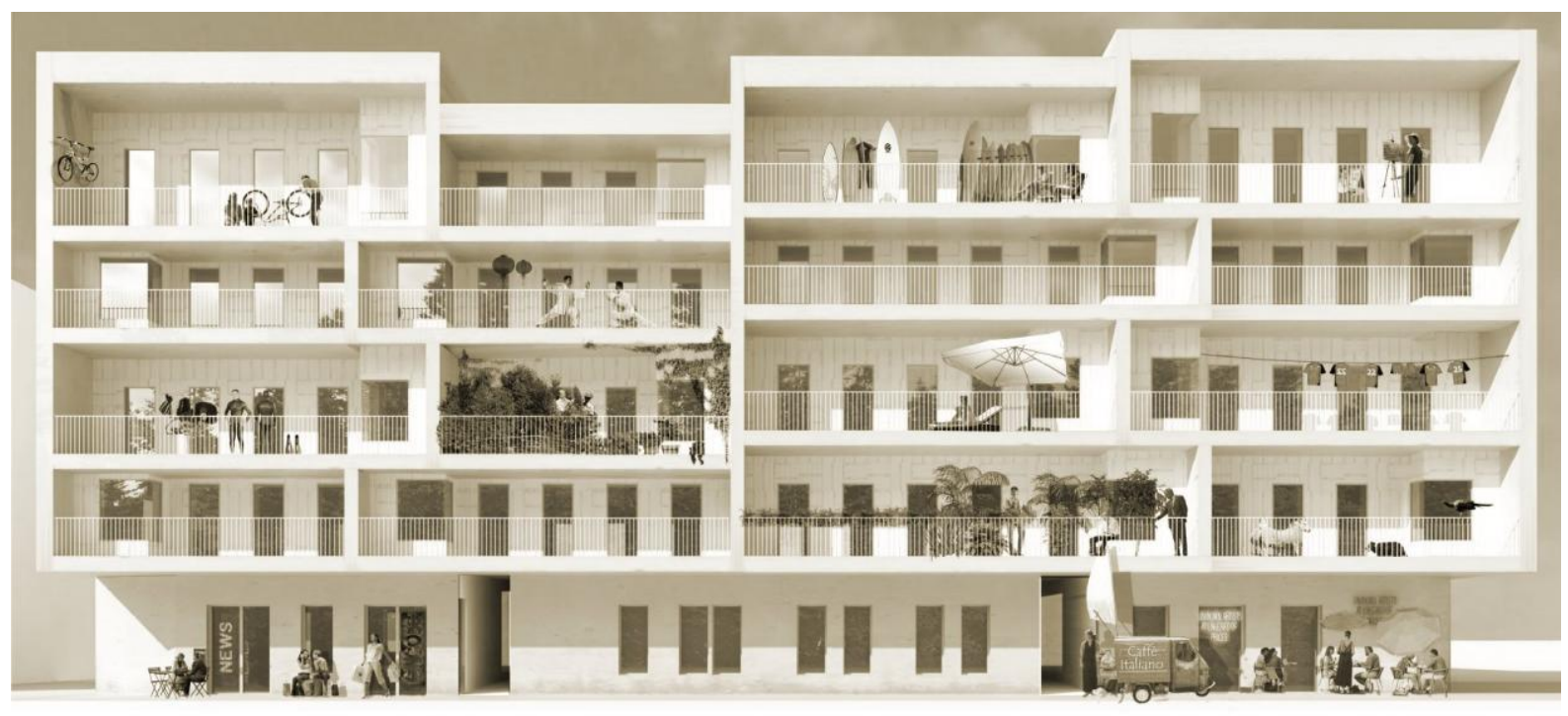

Figure 1 Koggens Gränd - Source: White Arkitekter

The researchers followed this project through its early stages, recording matters relating to 3D Design Coordination and collected further empirical data relating to requirements and procedures for this BIM-Use. In addition, common current practice methodologies were documented with a view to identify key issues to address. This case study enabled a thorough examination of the common issues hindering the work-flow of 3D Design Coordination and early Energy Analysis operations.

Hooper, M and Ekholm A (2012) 'A BIM-info delivery protocol', Australasian Journal of Construction Economics and Building, 12 (4) 39-52 


\section{Case study 2: KonsultHus - An Experimental Coordination Model}

Empirical data reflecting the results from this experimental model, which was developed and used by the author for this study, has enabled collection of further, more detailed information, eliminating unwanted variables, and facilitating a more precise examination of objects and properties required for 3D Design Coordination. 3D Design Coordination implies close collaboration and - on complicated projects - a frenzy of information exchange. For this reason it is critical to record and optimise information flow for this BIM-Use. Here it was examined what BIM information one needs to share with each other to allow for example:

4 the MEP consultant to proceed with design for plant requirements, ventilation duct sizing and routes, plumbing fixtures and pipe routes, drainage integration, incoming service routes and;

$\downarrow$ the Structural consultant to proceed with design for the foundations and structural frame.
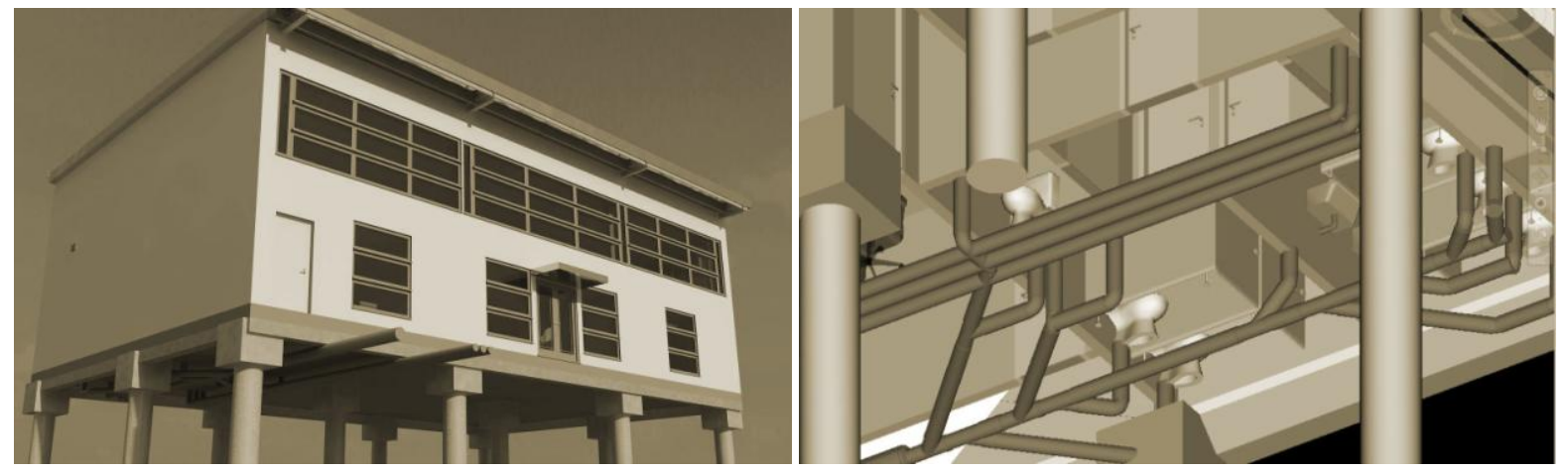

Figure 2 KonsultHus - An Experimental Coordination Model

It is clear that in order to optimise design information production and flow, parties must have at least a modest understanding of each other's information needs and, moreover; if you are not specific about the information you need, how can you be certain you'll get it?

Complaints of holding each other up because of lack of design information, or deliberately holding back design contributions until 'the architect has frozen the design', no longer washes with today's parallel BIM processes and collaborative design environment. Through this case study, an experimental exchange protocol was tested to help monitor the creation, distribution and timing of discipline BIM objects on a need-to-know basis.

\section{A State of the Art Review}

Many studies have been carried out to investigate, test and report on process development in the domain of BIM, not least information exchange and collaboration. Furthermore many studies still report difficulties and barriers both technical (such as interoperability) and nontechnical (such as organisational and team communication). Pazlar \& Turk (2008) for instance report 'data distortion and IFC interfaces not working as expected' whilst Pfitzner et al (2010) report on barriers relating to 'project organisation and commitment among project team members to collaborate'.

Much of existing research focuses on identifying existing barriers; this paper concentrates on developing a simple procedure that may help overcome certain communication barriers. Here in Sweden, it could be said that a particular obstacle to efficient information exchange in the context of BIM-Uses resides in under-developed national guidelines (Bygghandlingar 90) and the immaturities of the technical initiatives of the buildingSMART Alliance (including IFC, IDM's and MVD's) (Kiviniemi A. et al., 2008). 


\section{Bygghandlingar 90 \& Delivery Specifications}

This publication (Swedish Standards Institute, 2008), represents Sweden's chief guidelines for delivering digital information in connection with construction projects and is a valuable source of logical recommendations for managing building information in an organized and careful manner. However, it does not represent a BIM Standard and requires some development in a number of areas including that of BIM-Info Delivery Specifications. The output from this study aims to provide practitioners with a useable tool to address this deficiency and help the industry move towards procedural standardization.

\section{buildingSMART Alliance Initiatives}

Among the foundational standardization efforts of the buildingSMART alliance and its worldwide counterparts are the Information Delivery Manuals (IDMs) and Model View Definitions (MVDs) (buildingSMART, 2010). These are examples of the sector's collective recognition that better information is needed to support the development of better tools now emerging to deliver construction projects (Smith \& Tardif 2008). Technologies such as IDM and MVD are intended to help identify exactly what that information is by defining, for example, a model definition view for automated code checking and the information that must be included to generate that view. However, this is work in progress and is still a long way off being available for all DCO participants; as is the full capabilities of IFC (Pazlar \& Turk, 2008).

\section{Implementation}

\section{BIM-Info: A Consultant Perspective}

Whilst many consultants are demonstrating a strong interest in BIM there is a possible lack of practical knowledge in applying current technology and leveraging the much argued benefits of BIM. Other research (Gu \& London 2010) has revealed that DCO participant concerns primarily focus on practice, process and technically related issues. Here, through the events and discoveries revealed in particular through case study \#1, the following observations are highlighted:

$\downarrow$ Significant uncertainty exists amongst design team participant as to exactly what information to provide for each 3D Design Coordination Meeting.

$\downarrow$ Because of a lack of clarity, there was certain carelessness in providing quality BIM information.

$\leftrightarrow$ Quality checks on BIM information deliveries appear to be missing or inadequate prior to issue.

4 Apprehensions exist in delivering incomplete work or work in progress - suggesting a need for an additional BIM information status classification: WIP (work in progress).

$\downarrow$ Some design participants were reluctant to engage in design work and contribute to a developing design process prior to the Architects layout being 'frozen'.

$\downarrow$ Limited time/budget for design changes or iterations for specialist design participants - instead of productivity gains being fruitfully utilised to optimise the design, it presented an opportunity for some consultant organisations to simply take on more work.

$\rightarrow$ Among all participant disciplines, the architect was the most active member in attempting to resolve communication issues and align design team expectations in terms of information delivery and content requirements.

$\rightarrow$ Digital communication and information storage was established through a web portal to a project server. This enabled logging of all communications and a database for all current and live information.

$\rightarrow$ Folders were set up with associated access rights for each discipline enabling design participants to upload information in a commonly understood fashion. 


\section{Key Issues}

The emerging key issues that can be identified in connection with the above observations can be categorized and summarized as follows:

Practice issues:

$\downarrow$ Whilst project team members display enthusiasm and general interest in implementing BIM, there appears to be a lack of common understanding of what it entails not least in terms of BIM information deliverables.

$\downarrow$ Time commitment in the early stages presented difficulties and frustration, suggesting a resistance to change or flawed time planning.

$\downarrow$ Some localised competence issues in the use of 3D BIM authoring tools/lack of thoroughness in delivering quality information.

Process issues:

$\downarrow$ Willingness to collaborate and contribute towards the project design on the same information level (LOD) as others within the same timeframe was problematic, suggesting the need for stronger culture of BIM Implementation Planning together with a method of clearly articulating BIM information deliveries.

$\rightarrow$ Quality Control and validation of delivered BIM information was often left to the receiver to sort out - leading to down time for file clean-ups, deletion of duplicate objects etc.

Technical Issues:

$\rightarrow$ Naturally, not all design participants used to same BIM tools for model authoring. The transfer of MEP BIM information into the multi-disciplinary model for collision control presented problems. Here a lack of trust in data integrity emerged. The completeness and accuracy of 3D models remain a major concern for the design team particularly where models are exported from their native file formats.

\section{Difficulties}

In addition, derived from the case \#1 design team meeting observations and case \#2 practical experiments, a number of specific difficulties were identified relating specifically to 3D Design Coordination and early Energy Analysis.

3D Design Coordination - Common Problems:

$\downarrow 1000$ 's of collisions identified late in the design process with little opportunity to correct or solve them.

$\downarrow$ Early agreement on tolerance levels is critical including clearance between own discipline objects and other discipline objects.

$\downarrow$ Accountability for the maintenance and coordination of objects and properties must be clear.

$\downarrow$ Agreement of procedure for managing changes to the design required to mitigate or remove hard, clearance or duplication collisions.

$\uparrow$ Missing voids - Lack of accounting for voids for services including type, purpose, discipline, responsibility for correctness.

$\rightarrow$ Where objects are within the domain of both architect and structural engineer and in addition require input from the services engineers with regards holes etc, difficulties can arise through duplication. 
Energy Analysis - Common Problems:

$\downarrow$ No clear direction of what objects and properties are necessary for which analysis at what stage.

$\downarrow$ Tendency not to focus on authoring the right information at the right time.

$\downarrow$ Analysis carried out too late to have any pro-active impact on the design.

$\rightarrow$ Analysis results miss-interpreted.

$\rightarrow$ Analysis carried out by external consultant at a single point in time / results not acted on.

$\rightarrow$ No clear agreement or procedure for managing changes to the design to reduce energy consumption.

$\rightarrow$ Analysis carried out to confirm suspicions instead of to inform design and drive toward optimised solution.

$\rightarrow$ Major change in design instructed, focus on energy diminished or extinguished.

This evidence suggests that action is required to address these uncertainties and communication malfunctions. What is needed to combat these failures is clear and userfriendly articulation of process, what it involves and what information needs to be delivered by each party, when.

\section{BIM Information Deliveries}

As a valid alternative to the rather cumbersome and overly complicated MVD's being developed by the building SMART Alliance and others, this paper offers a simple method of defining, on a project basis, information deliveries for specific BIM Uses.

It has been suggested that if the industry is to move forward with BIM implementation, firms must focus on perfecting what they can deliver (Jernigan 2008). Initially this means reaching for the low hanging fruit such as 3D Design Coordination (through use of collision control tools) and early Energy Analysis (through use of built-in or associated energy simulation tools).

Both these BIM uses instantly add value to the DCO process and product and can be considered strategic, straightforward targets for consultant organisations to master in an efficient manner. What is problematic, however, is for team members to arrive at the same place at the same time with regards to BIM information quality and completeness. This is particularly critical in the context of the successful execution of various BIM-Uses including 3D Design Coordination. Project direction and information flow often meanders left and right of an efficient path resulting in frustration, loss of momentum behind value-adding processes, and often considerable time wasted.

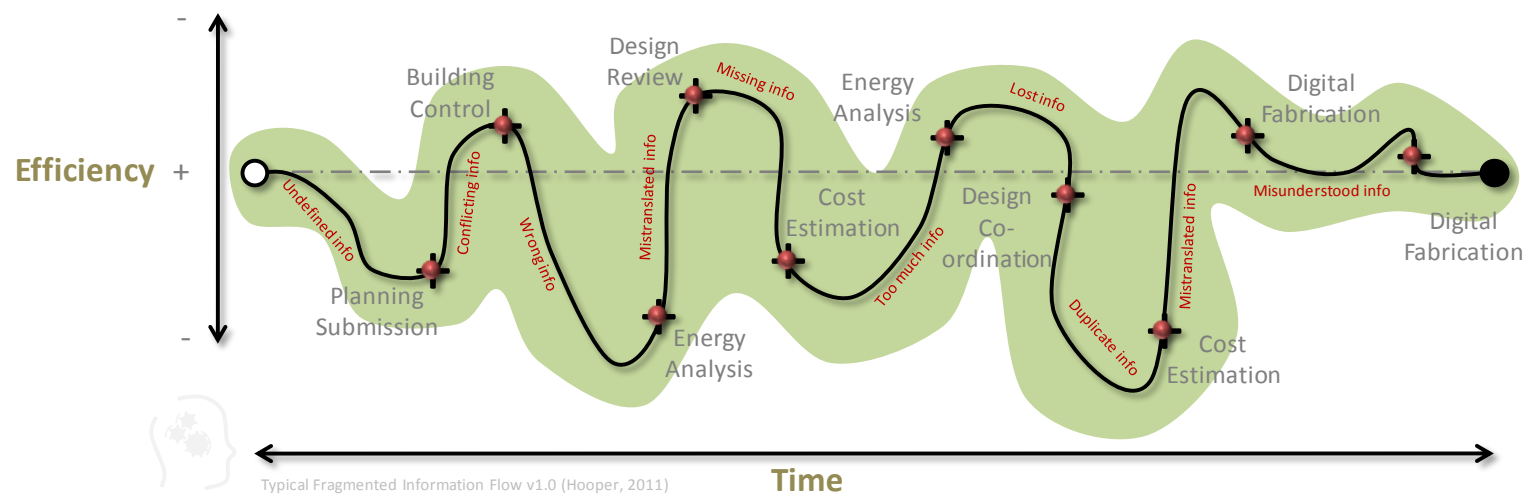

Figure 3 Information Flow - Traditional

Hooper, M and Ekholm A (2012) 'A BIM-info delivery protocol', Australasian Journal of Construction Economics and Building, 12 (4) 39-52 
Laying down considerable time and effort to carry out what should be routine tasks is a major concern to DCO players. Those who are bearing the pain of BIM implementation are struggling to leverage the benefits of added service and increased productivity as a result of downtime consumed by manually filtering, editing, adding, deleting, finding out if its valid and re-working building design information for what in theory should be sequential BIM Uses.

By encouraging all design participants to engage in work flow design and actively be aware of each other's information needs, the use of a BIM Information Protocol has the potential to straighten work flow and increase the accuracy and efficiency of information exchange. Coordination effort requires time but can be seen to lay the foundation for greater gains through the process.

Once organisations have succeeded and gained confidence in recording, purposefully designing and optimising their work-flows with attention to information exchange, project standards can develop into office standards and further to a National Standard. Within the sphere of information exchange, this study endeavours to organise and present key prerequisites necessary to set in motion a system design converging on standardisation.

So far this element of business practice within the AEC consultant sphere has been either largely missing or out of date. The figure below suggests a process which might help DCO participants move forward in earnest and articulating BIM information content with a view to optimising information flow and reducing exchange failures.

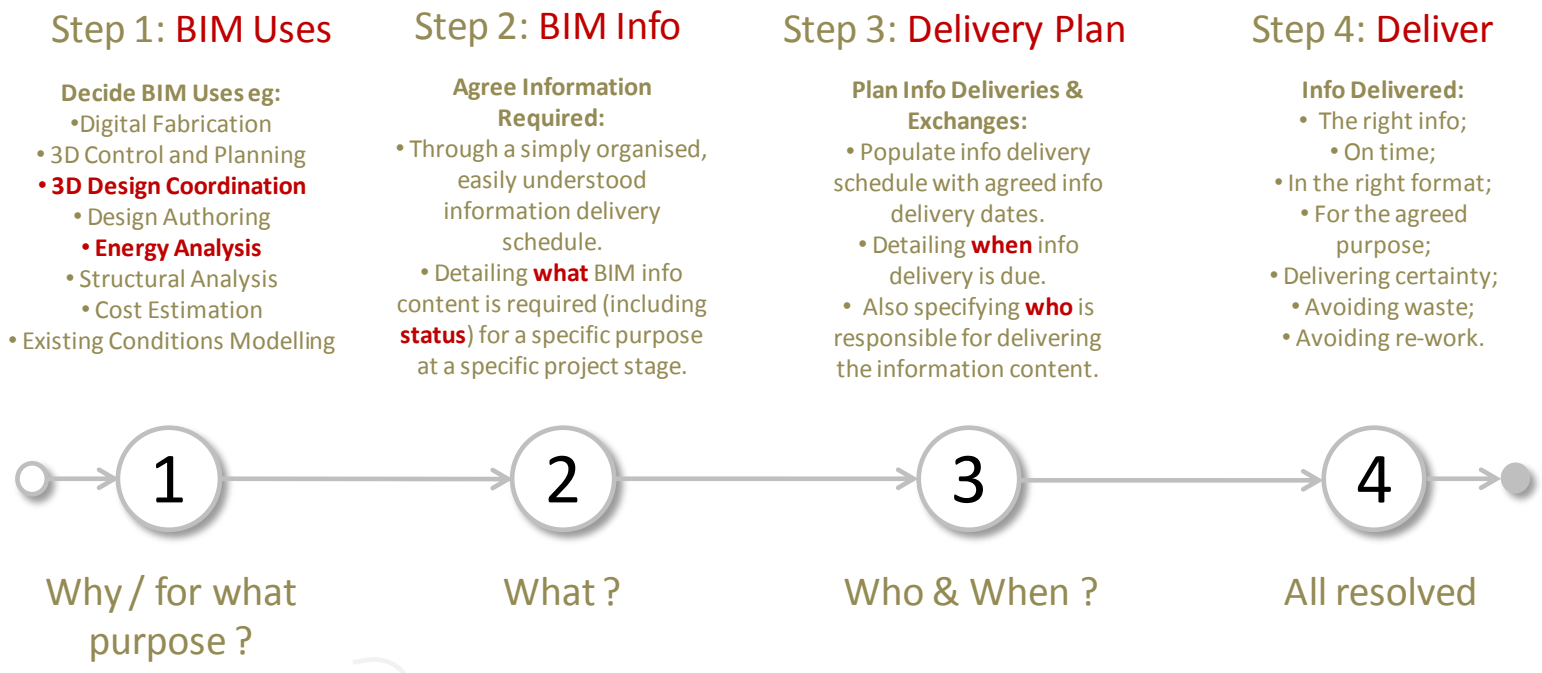

Figure 4 Strategic BIM-Info Delivery Process

\section{BIM-Info Delivery Protocol (IDP)}

Emerging from the observations and results revealed through discussions and experiments, the BIM-Info Delivery Protocol (IDP) is presented below as a sequence of pedagogical steps designed to respond to the research questions. 


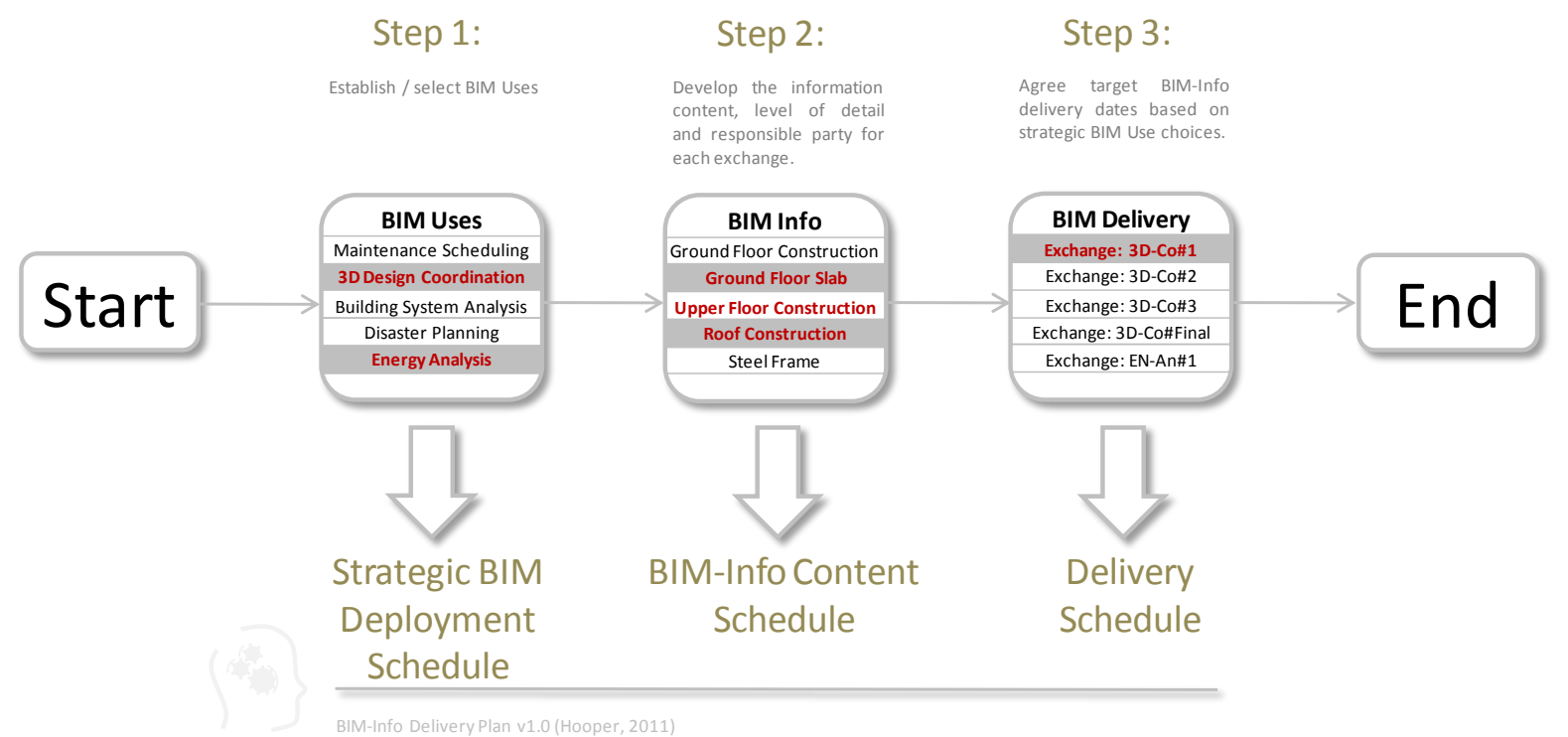

Figure 5 BIM-Info Delivery Protocol (IDP)

\section{Step 1: BIM-Uses}

The buildingSMART Alliance's Building Information Modelling Execution Planning Guide (Anumba et al., 2010) suggests a list of 25 typical BIM Uses including of course 3D Design Coordination and Energy Analysis. It naturally follows that teams must establish at the outset the strategic BIM-Uses they wish to deploy on a project specific basis. The decision to implement a BIM-Use must be based on resources, competency and anticipated value to the project (Anumba et al., 2010). Against each BIM-Use members should consider and articulate the timing of such activities through the BIM-Authoring stages to enable focus on imminent information demands and optimize information flow. The figure below illustrates how this might be articulated whilst enabling efficient implementation.

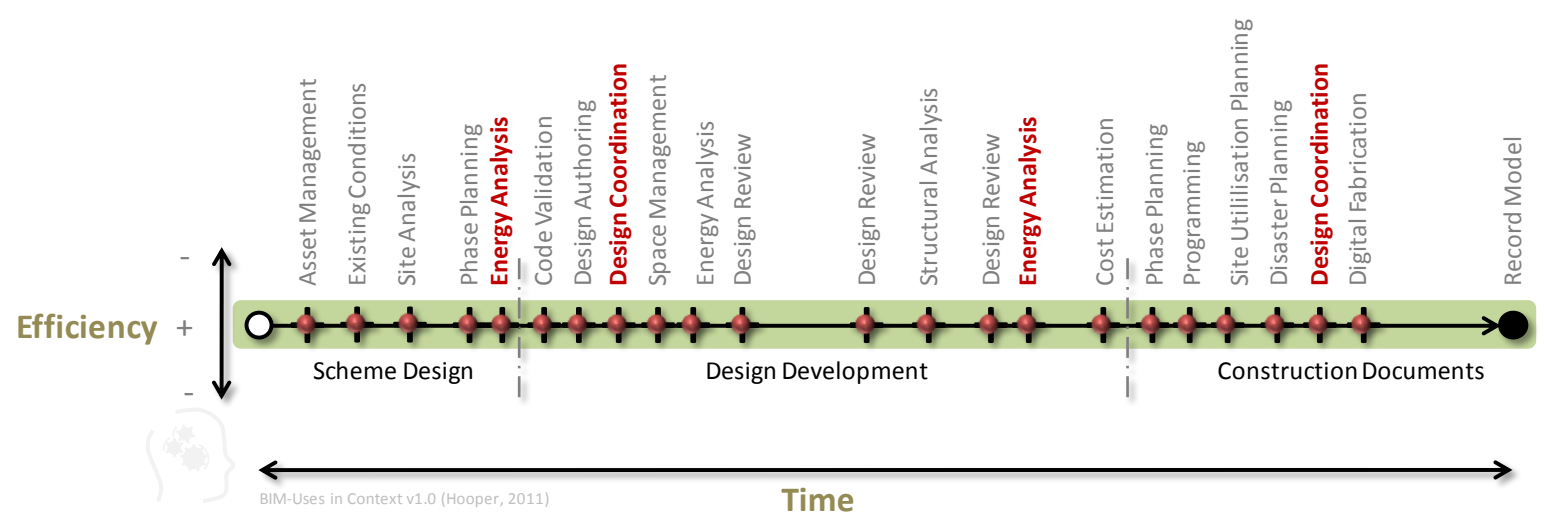

Figure 6 BIM-Uses in context

Often overlooked is the common business sense to the idea that, if certain design information can be supplied at right time, then its value to the project can be optimized. BIMUses should be selected for the right reasons - as drivers to the process and to help provide the data to support strategic decisions along the way. 


\section{Step 2: BIM-Info}

Here a definition of the model information content for strategic BIM implementation is articulated through scheduling the key objects with associated level of detail and responsible party. In this instance a BIM-Info Delivery Specification (IDS) template has been developed to express the information content and exchanges necessary to carry out and efficiently implement the BIM-Use: 3D-Co (3D Design Coordination) otherwise known as collision control.

The main software tools used to develop this schedule where Autodesk Revit Architecture together with Microsoft Excel. An important aspect of this study and resultant product is that by utilising industry standard tools and readily understood categories and classifications, consultants can maintain control of model content definitions and thereby build and retain trust in the exchange processes they create.

\section{Identifying BIM Objects \& Properties for Strategic BIM Uses}

The AIA (2008) amongst others has defined the concept of Levels of Detail (LOD) described through a sliding scale of LOD $100-500$ (Bedrick, 2008). In essence, the levels can be summaries as follows:

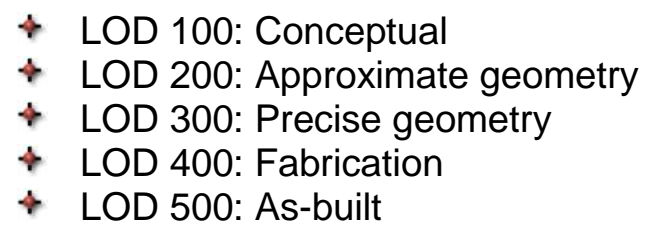

The LOD concept, established through the AIA's E202 Protocol published 2008 is now starting to be adopted throughout the world (Statsbygg, 2011). This standard together with an appropriate building element classification can be deployed to identify the BIM information required for specific tasks, however, in practice additional BIM objects and properties need to be identified out with the scope of most building classification systems. For this reason it is necessary to facilitate flexibility in BIM information scheduling and include, where appropriate, scope to articulate request for data such as:

Project Information
$\diamond$ Project Issue Date
$\diamond$ Project Status
$\diamond$ Info Status
$\diamond$ Client Name
$\diamond$ Project Address
$\uparrow$ Project Name
$\uparrow$ Project Number

\begin{tabular}{|c|}
\hline Project Units \\
\hline$\diamond$ Length \\
\hline$\diamond$ Area \\
\hline$\leftrightarrow$ Volume \\
\hline$\diamond$ Angle \\
\hline ^Slope \\
\hline$\diamond$ Currency \\
\hline
\end{tabular}

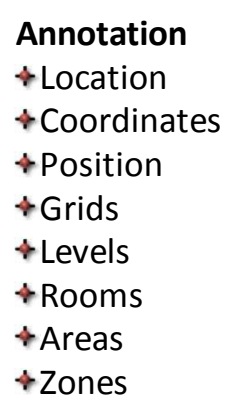

Other

$\diamond$ Voids

$\rightarrow$ Holes

The need for extra BIM object classifications beyond those to be found in national classification systems is clear. The above categories, identified through the authoring process of the KonsultHus case project, have been included in the BIM-Info Delivery Specification to enable transfer of that information between consultant disciplines in a clear and comprehensive way. 


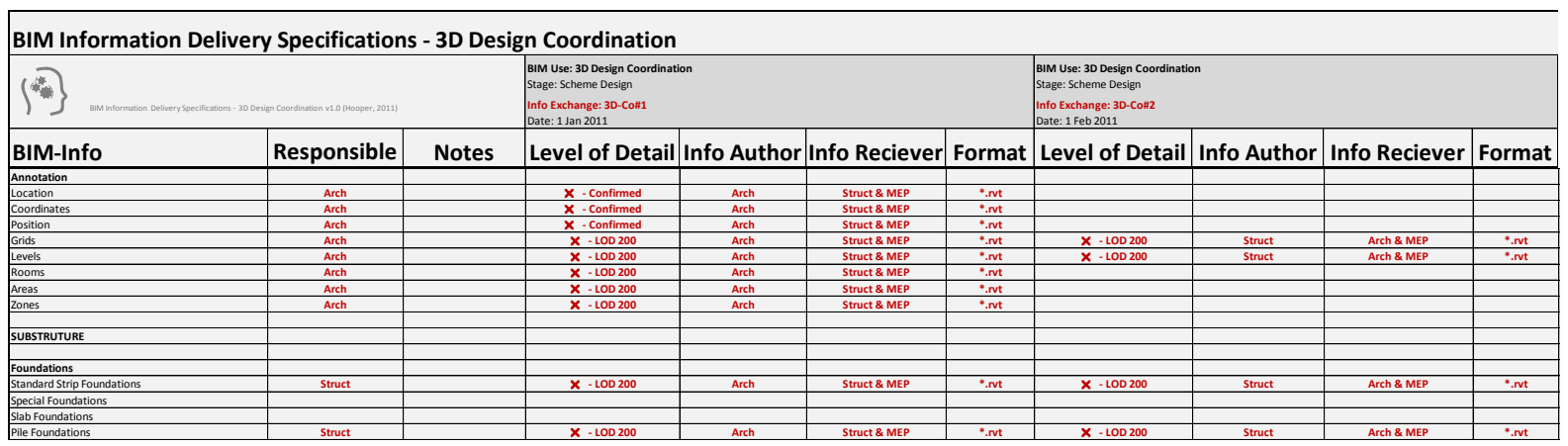

Figure 7 Extract from BIM-Info Delivery Specifications (IDS)

An innovative feature, still under development, is the possibility of automating the extraction of requested BIM information through an XML schema, enable through the IDS spreadsheet, thus eliminating manual filtering and if perfected, scope for error. A prerequisite of this novelty is a thorough and complete tagging of objects to the utilized building components classification.

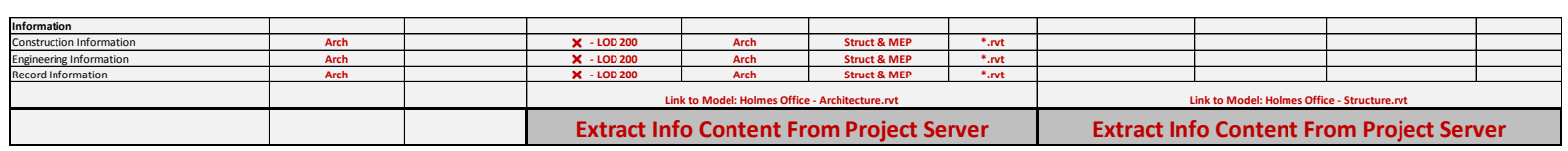

Figure 8 BIM Information Content Extraction

\section{Step 3: BIM-Delivery}

Registry of BIM information exchanges can be readily recorded and communicated through project networks in accordance with the delivery schedule. However, often neglected is a sub-process of quality control. This is necessary more than ever, not only to demonstrate due diligence, but also to ensure the content of the BIM information deliveries match with the general expectations of the project team as articulated in the BIM-Info Delivery Specification schedule. This process is essential to eliminate rework for receivers and endeavours to place the onus on the supplier to ensure the contents is what it says it is.

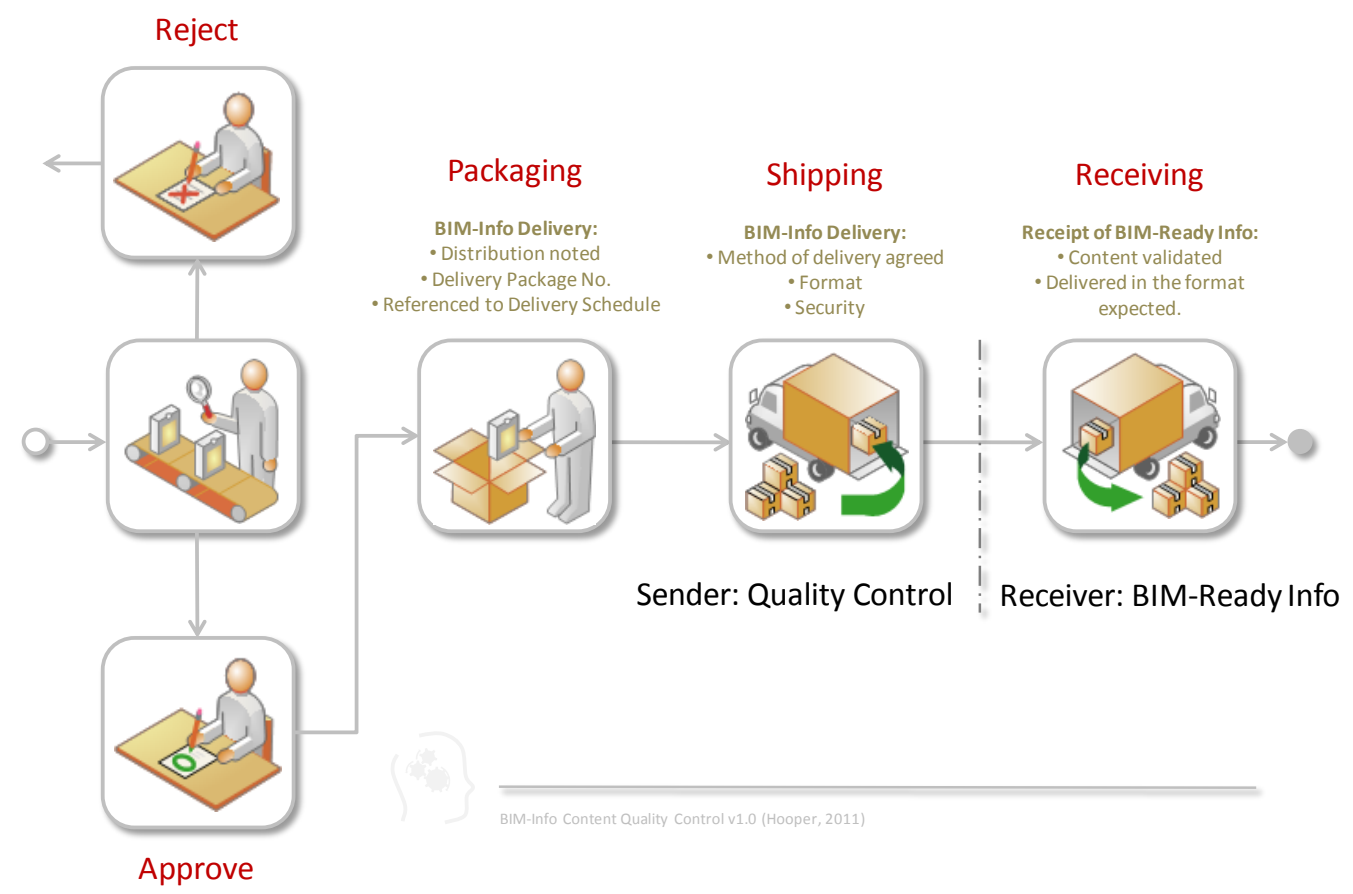

Figure 9 BIM-Info Content Quality Control Measures

Hooper, M and Ekholm A (2012) 'A BIM-info delivery protocol', Australasian Journal of Construction Economics and Building, 12 (4) 39-52 


\section{Conclusions}

\section{Summary of Main Findings}

The key deliverable from this study is the BIM-Info Delivery Protocol (IDP) which attempts, through use of straight-forward and easy to use tools, to align consultant BIM information delivery expectations and represents a development of the concept behind Leveransspecifikationer mentioned but not substantiated in Bygghandlingar 90. By recording information flow properly we can better understand each other's information needs and reduce the risk for misunderstanding. If handled optimally BIM information can significantly enhance the quality of the product and safeguard the success of project. A clear and commonly understood picture of the BIM information deliveries through establishing a project standard BIM-Info Delivery Specification offers a tangible solution to help consultant disciplines manage BIM information.

To add maximum value to the project, the timing and content of BIM authorship is critical. By articulating planned BIM-Uses, the necessary BIM information needed to carry out these Uses together with target BIM-Delivery dates; project teams can more readily focus on the strategic task in hand and help each other to deliver the intended result in an efficient manner.

The BIM-Info Delivery Protocol (IDP) is a compelling tool for use in the evolving world of virtual design and construction teams and can be used as a basis for a BIM Management Plan. However, industry reference-group feedback has suggested a number of limiting factors including that "it would be an additional burden, indeed laborious, to fill out the IDS when projects are already on a tight time schedule and budget. If teams cannot directly see the positive effects of using such a protocol, it may be difficult to achieve widespread uptake." This protocol represents a tool for improvement, a first step could be to first record one's own strategic information requirements, recognition of this together with patience may prove to be prerequisites.

\section{Positioning of BIM-Info Delivery Protocol (IDP) v. IDM \& MVD}

On reflecting on the positioning of the BIM-Info Delivery Protocol in relation to IDM and MVD a number of discrete characteristics can be identified:

$\downarrow$ The IDP represents a ready-to-use tool for communicating and aligning information exchange expectations, independent of software application.

^ The development of IDM and MVD's have yet to reach maturity and are dependent upon the complete and successful implementation of the IFC model within the BIM authoring software applications (NIBS, 2007) - this has yet to be realised and there is resistance within a number of the key software suppliers to do so.

$\rightarrow$ The principle difference in methodology between the IDP and buildingSMART's IDM/MVD is that buildingSMART's purpose is to solve interoperability. Whilst this is a crucial goal, the IDP method is needed as a first practical step. User results can serve as useful input to the ongoing work with IDM's and MVD's.

$\downarrow$ The IDP is orientated towards the Architects and Engineers who represent the key members of the model authoring team.

\& Whilst IDM \& MVD require in-depth technical IT and systems knowledge, often outside the scope of expertise of many DCO project participants, the IDP may provide the industry with an easy to use, working alternative to IDM \& MVD that can be readily developed in-house and re-used for future projects.

\section{The Need for a Common Method of Defining BIM deliverables}

One of the principle difficulties in realising efficiency gains through the use of BIM is a function of defective communication stemming from, amongst other things, a general lack of standard terminology and methods of describing process and deliverables. 
In the absence of existing simple standard methods of defining the content of BIM information exchanges the BIM-Info Delivery Protocol attempts to fill this gap. Furthermore, beyond aligning information needs and creating greater certainty through intelligent and value-adding deliverables, the final built product will have an increased propensity to be as expected.

\section{Summary of the Key Features of IDS}

The BIM-Info Delivery Specification seeks to provide a simple standard method of describing information exchange content. As a decision-support tool, its key elements can be readily identified per the illustration below.

Through the use of these generally understood and commonly recognised concepts, brought together in a standard way, the IDS can help enable succinct communication of content requirements for key information exchanges. At the core of this process is defining, BIMUse, Information Exchange (No. within the series), Delivery Date, Responsible Party, Level of Detail, Receiving Party, and Delivery Format.

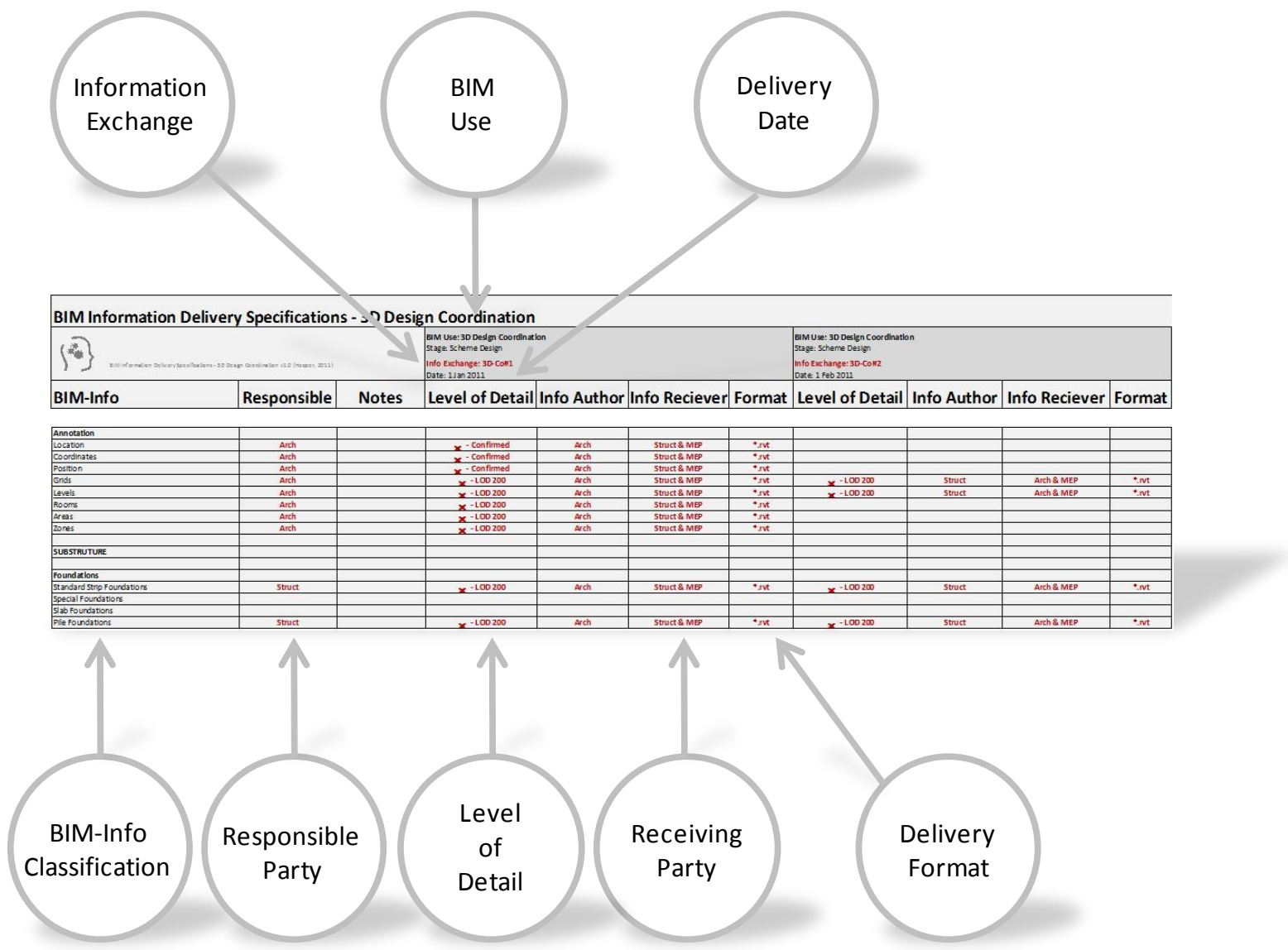

BIM-Info Delivery Specification v1.0 (Hooper, 2011)

Figure 10 BIM-Info Delivery Specification - Constituent Parts

\section{Further Research}

It may prove possible to generate machine-readable model information content definitions through the XML schema making it possible to standardise such contents and deliver project information through automated processes.

Hooper, M and Ekholm A (2012) 'A BIM-info delivery protocol', Australasian Journal of Construction Economics and Building, 12 (4) 39-52 


\section{Acknowledgements}

Particular thanks to Gunilla Qvarnström of White Architects and the Koggens Gränd Project Team. This research project is funded by SBUF, Formas-BIC, Interreg VI and members of OpenBIM.

\section{References}

AIA (2008) AIA Document E202-2008: Building Information Modeling Protocol Exhibit. AIA and AIA California Council, California, USA

Anumba, C., Dubler, C., Goodman, S., Kasprzak, C., Kreider, R., Messner, J., Saluja, C. and Zikic, N. (2010) Building Information Modelling Project Execution Planning Guide, Version 2.0, The Computer Integrated Construction Research Group, The Pennsylvania State University, Pennsylvania, USA

Bedrick, J. (2008) Organizing the Development of a Building Information Model, AECBytes, http://www.AECBytes.com, visited Jan 2010

BuildingSMART (2010) http://www.buildingsmart.com, visited Jan 2010

CRC Construction Innovation (2009) National Guidelines for Digital Modelling, Cooperative Research Centre for Construction Innovation, Brisbane, Australia

Ekholm A., Häggström L., Johansson B., Tarandi V. and Tyrefors B. (2010) RoadMap för digital information om byggd miljö, Lund University, Lund, Sweden

$\mathrm{Gu}, \mathrm{N}$. and London, K. (2010) 'Understanding and facilitating BIM adoption in the AEC industry', Automation in Construction, 19, 988-999

Jernigan, F. (2008) BIG BIM little bim: The Practical Approach to Building Information Modeling-integrated Practice Done the Right Way!, 4 Site Press, Salisbury

Kiviniemi A., Tarandi V., Karlshöj J., Bell H. And Karud, O. (2008) Review of the Development and Implementation of IFC Compatible BIM, Final report of the Erabuild project, Erabuild

NIBS (2007) National Building Information Modeling Standard - Version - Part 1 - Overview, Principles, and Methodologies, NBIMS, USA: National Institute of Building Sciences

Pazlar, T. and Turk, Z. (2008) 'Interoperability in Practice: Geometric Data Exchange Using the IFC Standard', ITCon, 13, http://itcon.org/data/works/att/2008 24.content.00881.pdf, visited Mar 2010

Pfitzner, M., Benning, P., Tulke, J., Outters, N., Nummelin, O. and Fies, B. (2010) InPro D29 - Barriers and Opportunities - Future ICT and Organisational Requirements, The InPro Consortium

Robson, C. (2002) Real world research : A resource for social scientists and practitionerresearchers, Blackwell, Oxford

Smith, D and Tardif, M. (2009) Building Information Modeling: A Strategic Implementation Guide for Architects, Engineers, Constructors, and Real Estate Asset Managers, John Wiley \& Sons, New Jersey

Statsbygg (2011) BIM-Manual 1.2: Statsbygg Building Information Modeling Manual version 1.2 (Draft), Statsbygg, Oslo

SIS (2008) Bygghandlingar 90 : byggsektorns rekommendationer för redovisning av byggprojekt. D. 8, Digitala leveranser för bygg och förvaltning, SIS Förlag AB, Stockholm

Yin, R. K. (2003) Case Study Research: Design and Methods (Applied Social Research Methods), Sage Publications, London 